\title{
A Low-Profile Beam-Steering Reflectarray with Integrated Leaky-Wave Feed and 2-Bit Phase Resolution for Ka-band SatCom
}

\author{
Qiaoshan Zhang, Mingtao Zhang, Xiaowei Shi, Steven Gao, Fellow, IEEE, \\ Qi Luo, Senior Member, IEEE, Lei Chen, Jixiang Wan and Xudong Wang
}

\begin{abstract}
A novel reflectarray (RA) with ultra-low-profile and 2-bit phase quantization beam-steering ability is presented in this paper. To reduce the profile, a Leaky-wave feed is used to excite the RA with enhanced illumination efficiency. Moreover, simultaneous sum and difference patterns are also obtained to provide beam flexibility. The entire thickness of the proposed RA is less than $3 \%$ of that of the conventional front-fed RA with the same aperture. To increase the efficiency of the RA, a novel unit cell consisting of a polarizer layer and a reflection layer is developed, which is configured to provide polarization rotation and 2-bit phase shifts by using a hybrid of tunable polarization and discrete resonator. The operation principle, theoretical explanation, and implementation of the proposed antenna are elaborated in this work. To prove the design concept and beam scanning performance, an array with $9 \times 7$ unit cells operating at Ka-band is designed and simulated firstly. 2-D beam scanning within the range of $\pm 30^{\circ}$ has been verified. Then, a passive prototype with $9 \times 67$ unit cells is designed, fabricated and measured. Experimental results show aperture efficiency of $35.1 \%$ and illumination efficiency of $43.4 \%$. The developed RA is scalable, and it provides a viable low-cost solution to develop low-profile, high-gain and beam-steering array antennas for satellite applications.
\end{abstract}

Index Terms-reflectarray, low-profile, beam-steering, 2-bit phase resolution, sum/difference beams, leaky-wave feed.

\section{INTRODUCTION}

$\mathrm{P}$ rinted microstrip reflectarrays (RAs) have been widely used in modern wireless applications as solutions of high gain and beam-scanning antennas. They combine some of the advantages of conventional reflector antennas and phased arrays [1]. Instead of using the parabolic reflector, a lightweight planar structure is applied to reduce the fabrication complexity and volume. In addition, the bulky, lossy and costly beamforming networks of the phased array are avoided by using a spatially feeding mechanism. Hence, the RA shows

This work was supported by the China Aerospace Science and Technology Corporation (CAST), and in part by scholarship from the China Scholarship Council and EPSRC grants EP/N032497/1 and EP/S005625/1 (Corresponding author: Qiaoshan Zhang)

Q.-S. Zhang, X.-W. Shi and L. Chen are with Xidian University, Xi'an 710071, China. (e-mail: zhangqs_504@163.com).

S. Gao is with the School of Engineering and Digital Arts, University of Kent, Canterbury CT2 7NT, U.K.

Q. Luo is with the School of Physics, Engineering \& Computer Science, University of Hertfordshire, Hatfield, Hertfordshire, AL10 9AB, U.K.

M.-T Zhang, J.-X. Wan and X.-D. Wang are with the Xi'an Institute of Space Radio Technology, Xi'an 710100, China. potential superiority as an ideal choice for mobile satellite communication (SatCom) systems. However, one drawback of the RA for such applications is the high profile due to the spatially feeding configuration.

Low profile is a highly desirable feature of RA in applications that require low aerodynamic drag or low space consumption, such as satellites and radars. The conventional RA is configured with the array plane illuminated by a feed such as a horn, as shown in Fig. 1 (a), and the height is proportional to the aperture size. To reduce the height to half of that of the conventional front-fed RA, the Folded Reflectarray Antennas (FRAs) were proposed in the 2000s [2] and developed in literature [3]-[7], as the configuration shown in Fig. 1 (b). In [8], a leaky-wave structure was employed as the feed to further reduce the profile of the RA. This configuration can break the correlation between the focal length and the aperture size, and then realize the low-profile purpose especially for large-scale arrays. However, only limited simulation works are shown and there is a lack of feasible models of excitation methods for the leaky-wave structure. In [9], a waveguide leaky-wave cavity excited by a taper is applied as a feed for a transmitarray (TA), but this design can't be extended to apply for a RA and the bulky taper is of low efficiency. Thus, there is a technology gap of an efficient leaky-wave feed for the RA.

On the other hand, various reconfigurable technologies have been introduced to gain the beam-steering ability and these techniques are summarized in [10]. Among them, the discrete phase shift method especially 1-bit phase resolution is preferred for better linearity, easier control and lower power consumption [11]-[13]. Nevertheless, large phase compensation errors caused by the 1-bit phase quantization leads to reduced gain and scanning performance. The directivity of a 2-bit array is approximately $3 \mathrm{~dB}$ higher than that of a 1-bit array [14]. Thus, some works on 2-bit phase shifter technologies have been presented in [15]-[18]. Yet, these works focus solely on the unit cell design controlled by embedded lumped elements to achieve discrete resonances with respect to different equivalent electrical lengths. As a result, limited implementations of 2-bit beam-steering RA have been reported because of the system complexity caused by lumped elements and control circuits. To the authors' best knowledge, the demonstration of 2-bit RA with the above discretely resonant elements can be only found in [19] and [20]. In [21], the polarization-rotation concept is introduced in a RA unit cell to realize 2-bit phase shifts by 

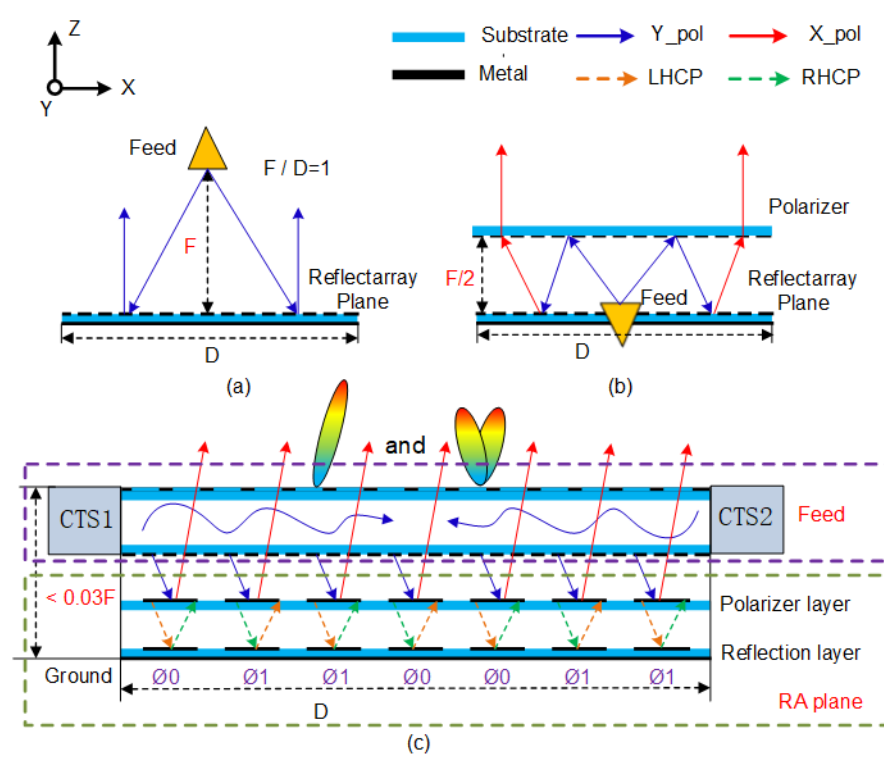

Fig. 1. Configurations and operating principles of several reflectarrays with the aperture of $39.2 \lambda_{0} \times 5.4 \lambda_{0}$. (a) Conventional front-fed reflectarray. (b) Folded reflectarray. (c) The proposed reflectarray.

using mixed polarization-rotation (PR) and non-PR operations. However, there is no reported design on a polarization-rotation RA unit cell based on the concept of the linear-to-circular polarization conversion. Although the circular polarizer has been widely used in RAs for the conversion [22-23], it is utilized to realize phase shift in a RA unit cell for the first time.

In this paper, a novel architecture of a low-profile 2-bit beam-steering RA with integrated CTS-leaky-wave feed is proposed, and the schematic diagrams and configuration is shown in Fig.1 (c). Assuring the ratio of focal length and diameter in conventional architecture is equal to 1 , the entire thickness of the proposed RA is lower than 3\% of that of the conventional one, and the thickness will keep constant as the array gets larger. Inspired by the leaky-wave antenna [24]-[25] and the continuous transverse stub (CTS) antenna [26]-[28], a new configuration of a leaky-wave structure excited by opposite placed CTS arrays is developed. Moreover, the sum and difference beams can be obtained simultaneously with the two CTSs excited by in-phase and out-of-phase fields from a waveguide magic- $T$.

In the RA design, a new unit cell operating at Ka-band is presented with 2-bit phase quantization and polarization rotation for the first time. A combined approach of tunable polarization and discrete resonator is applied to realize the 2-bit phase shift. The unit cell is designed to provide four phase states that are electronically controlled by the embedded MEMS elements. Full-wave simulation of the active unit cell shows wide phase bandwidth and phase linearity. Moreover, several passive RAs with fixed beam angles are simulated, showing $\pm 30^{\circ}$ beam-steering angular range at both E-plane and $\mathrm{H}$-plane with gain fluctuation lower than $3 \mathrm{~dB}$. A wide gain bandwidth with less than $0.25 \mathrm{~dB}$ gain variation is obtained from $28 \mathrm{GHz}$ to $32 \mathrm{GHz}$. The impedance bandwidth of the presented RA is about $3 \%(29.5 \sim 30.5 \mathrm{GHz})$, which is suffered

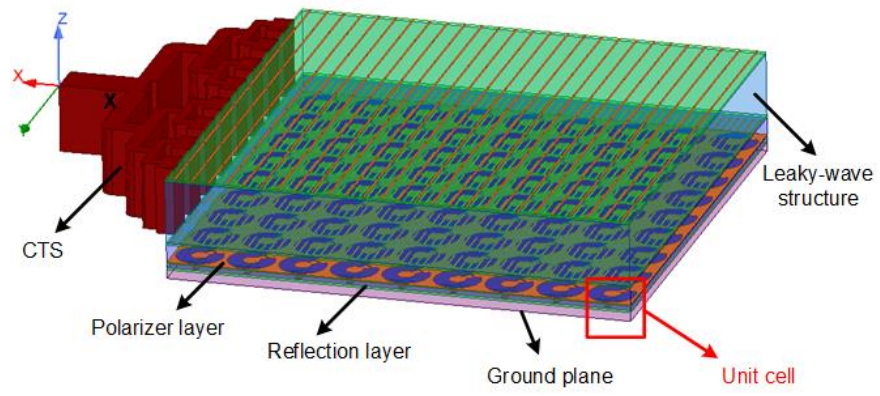

Fig. 2. 3-D model of the proposed reflectarray (Half part of the array).

from the narrow bandwidth of the unit cell and it can be improved by the optimization of the unit cell. To validate the design, a passive RA prototype consisting of $9 \times 67$ unit cells with the main beam at boresight is simulated, fabricated and measured. Experimental results show an aperture efficiency of $35.1 \%$ and a low cross-polarization level of $-28.2 \mathrm{~dB}$. Although the sidelobe levels (SLL) are higher than the conventional RA due to the inverse-taper magnitude distribution on the feed aperture, the illumination efficiency of the feed is equal to $43.4 \%$, which is relatively high for the large-scale array with an extremely low profile. With regard to the difference beam, the measured null-depth is higher than $24.7 \mathrm{~dB}$. All the above features show that the proposed RA is an attractive candidate for the satellite antennas with high efficiency, low profile and flexible beams.

The paper is organized as follows. The operation principles of the low profile and 2-bit phase resolution are introduced and analyzed in Section II. Based on these principles, the design of the unit cell and CTS-leaky-wave feed are described in Section III. The array performance analysis and the description of the RA design are shown in Section IV. The experimental results of the prototype are given and analyzed in Section V. Finally, Section VI concludes this paper.

\section{PRINCIPLES OF THE REFLeCtARRAY WITH INTEGRATED CTS-LEAKY-WAVE FEED AND 2-BIT Phase ResOlution}

\section{A. Configuration and Operation Principle}

The schematic of the proposed RA and the electromagnetic (EM) wave propagating paths are shown in Fig. 1 (c). Similar to the FRA, the incident wave is linearly polarized, while the radiating wave is orthogonal in the polarization. The limitation of the proposed architecture is that it only operates at single linear polarization. If circular polarization is required, it can be realized by a polarizer located on the top of the whole reflectarray [5]. In the RA, the leaky-wave structure has two functions: one is to serve as a feed, and the other is to operate as a polarizer to distinguish the incident and radiating waves. In addition, the RA plane is required to rotate the polarization. Consequently, the operation principle can be described in detail. Firstly, the y-polarized wave from the leaky-wave feed leaks into the polarizer layer and is converted into a circular polarization (CP) wave. Then, the polarization of this $\mathrm{CP}$ wave is twisted by the reflection layer due to the reverse direction of 

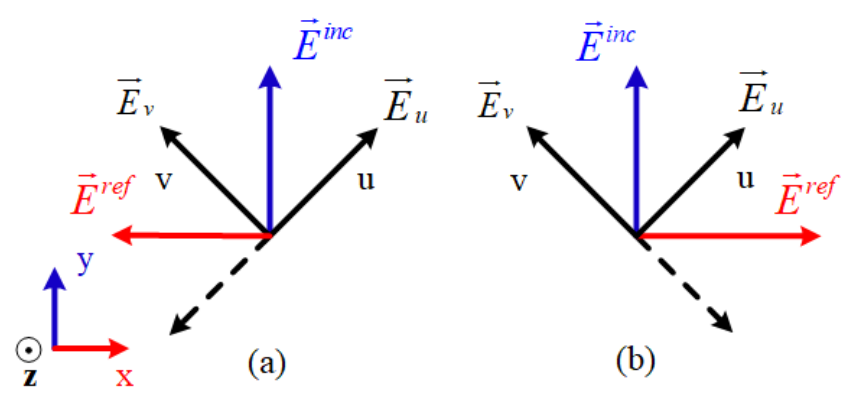

Fig. 3. Schematic diagrams of polarization rotation concept with different polarizer design. (a) LHCP. (b) RHCP. Superscripts "inc" and "ref" refers to the incident and reflected field.

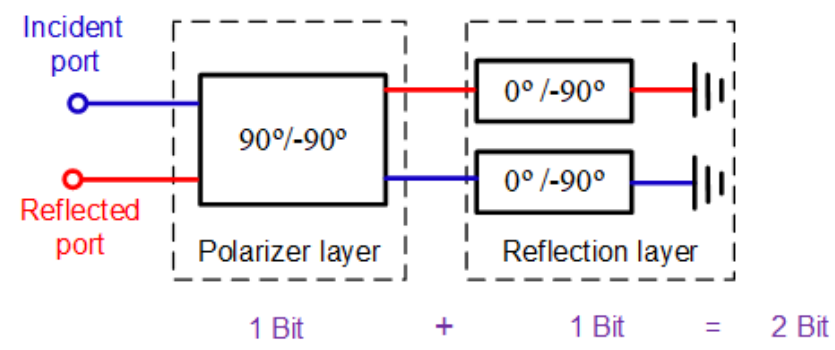

Fig. 4. Schematic diagram of the proposed 2-bit phase quantization concept.

the EM wave propagation. Finally, the orthogonal $\mathrm{CP}$ wave can be converted into the x-polarized LP wave by the polarizer layer, which then pass through the leaky-wave structure and radiate to the free space.

The 3-D model and configuration of the presented antenna is shown in Fig.2, and it can be seen that the CTS-leaky-wave feed is suspended directly above the array of 2-bit unit cells for the reason that the leaking wave can excite the array through the leaky mode coupling. Thus, the entire thickness of RA can be greatly reduced by leaky-mode control rather than the $f / D$ spacing-feeding constraint in conventional RA and reflector antennas, forming a low-profile mechanism.

\section{B. Principle of Polarization Rotation and 2-bit Phase shifts}

To realize the polarization rotation of the incident electric field, the use of rectangular patches with axes tilted by $45^{\circ}$ to the incident LP electric field is a classic approach [2], [11]. Dual-polarized elements with two polarized ports connected are reported [3-4]. The way to realize the polarization rotation in our design is different from the above methods. When the incident LP wave impinges on the polarizer, it is converted to left-handed or right-handed circular polarization (LHCP or RHCP) wave. Assuming the coordinate system in Fig. 3 is based on the top view of the RA, and the polarizer is an LHCP design, therefore, the incident field $\left(\vec{E}^{i n c}=\hat{y} E_{0} e^{j k z}\right)$ is decomposed into two orthogonal components $\left(\vec{E}_{u}, \vec{E}_{v}\right)$ with $90^{\circ}$ phase difference after passing through the polarizer. The LHCP wave can be described as

$$
\vec{E}_{L H C P}^{i n c}=\hat{y} E_{0} e^{j k z}=\frac{E_{0}}{\sqrt{2}} e^{j k z}(\hat{u}+j \hat{v})
$$

Then, the reflected wave from the reflection layer is

$$
\vec{E}_{R H C P}^{r e f}=-\frac{E_{0}}{\sqrt{2}} e^{j(-k z+\Delta \varphi)}(\hat{u}+j \hat{v})
$$

where $\Delta \varphi$ is the phase compensation controlled by the reflection layer. The final radiated wave after transmitting from the polarizer layer is calculated as

$$
\begin{aligned}
\vec{E}^{r a d} & =-\frac{E_{0}}{\sqrt{2}} e^{j(-k z+\Delta \varphi)}\left(\hat{u}+j e^{\frac{j \pi}{2}} \hat{v}\right) \\
& =\frac{E_{0}}{\sqrt{2}} e^{j(-k z+\Delta \varphi)}(\hat{v}-\hat{u})
\end{aligned}
$$

This is an LP wave in x-polarization along the z-direction. Therefore, the incident y-polarized wave is rotated into an $\mathrm{x}$-polarized wave pointing to $-\mathrm{x}$-direction. Similarly, if the polarizer is an RHCP design, the radiated wave will be an LP wave of $\mathrm{x}$-direction, as shown in Fig. 3 (b). Consequently, $90^{\circ} /-90^{\circ}$ phase shifts can be obtained as 1-bit phase quantization by controlling the polarization direction of the polarizer layer. Moreover, the reflection layer can provide another 1-bit phase shift with $90^{\circ}$ difference for $\Delta \varphi$. Therefore, four phase states of $-90^{\circ} / 0^{\circ} / 90^{\circ} / 180^{\circ}$ are achieved. In other words, the proposed unit cell is equivalent to a 3-D reflection-type phase shifter with a combination of 1-bit discrete resonator phase shifter and 1-bit tunable polarization phase shifter to realize the 2-bit phase quantization, as the schematic diagram shown in Fig. 4.

\section{Design of Phasing Unit Cell and Low-Profile Feed}

\section{A. Unit Cell for the Reflectarray}

The unit cell is a multi-layer printed circuit board (PCB) structure consisting of a polarizer layer, a reflection layer and a ground plane. The Rogers 5880 with a dielectric constant of 2.2 and loss tangent of 0.0009 is selected as the dielectric substrates for the polarizer and reflection layers with the same thickness of $0.127 \mathrm{~mm}$. In addition, the gaps between different layers are fulfilled with thick Rohacell foams as structural support, whose dielectric constant and loss tangent is 1.06 and 0.0017, respectively. The configuration of the unit cell is illustrated in Fig. 5 (a), and the geometrical parameters are listed in Table. I. The unit cell is $0.59 \lambda_{0} \times 0.59 \lambda_{0}$ at the center frequency $f_{0}=30 \mathrm{GHz}$ to avoid grating lobes in the $\pm 30^{\circ}$ beam scanning range.

As shown in Fig. 5 (b), the polarizer layer is designed with a new patch pattern on a single-layer PCB. The pattern is a defected split ring slot, and two sector metal links with ring slots between the inner and outer metal are symmetrically located in both sides of the $\mathrm{x}$-axial. The ring slot is capacitive for the vertical component of the incident electric field with respect to the slot direction, which is inductive for the horizontal component. The transmission phase of the inductive component lags behind the capacitive component. By changing the slot gap $(s)$ and the sector angle (ang), the equivalent capacitive reactance and inductive reactance can be optimized to achieve $90^{\circ}$ phase difference of both components. Two MEMS switches are integrated into the ring slot to switch the RHCP and LHCP conversions for the polarizer. When the two 


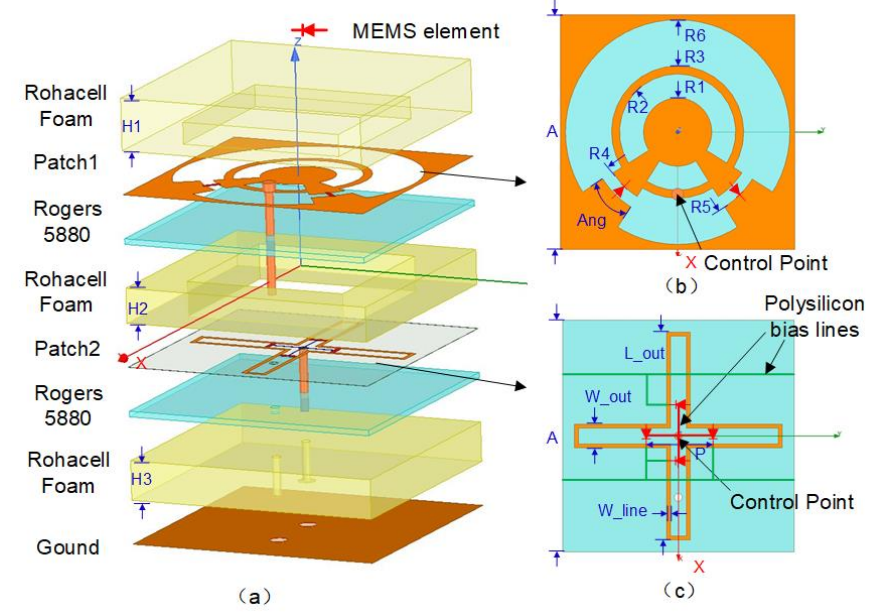

Fig. 5. Configuration and geometry of the proposed 2-bit unit cell. (a) 3D-view. (b) top-view of the polarizer patch. (c) top-view of reflection patch.

TABLE I

DIMENSION OF THE UNIT CELL $(\mathrm{mm})$

\begin{tabular}{|c|c|c|c|c|c|c|c|}
\hline A & R1 & R2 & R3 & R4 & R5 & R6 & Ang \\
\hline 5.85 & 0.85 & 1.45 & 1.65 & 1.9 & 2.2 & 2.8 & $25.6^{\circ}$ \\
\hline L_out & W_line & W_out & P & H1 & H2 & H3 & \\
\hline 5.25 & 0.1 & 0.6 & 1.5 & 1.2 & 0.8 & 0.9 & \\
\hline
\end{tabular}

MEMS elements show state \{"off", "on"\} from the left to the right, the right slot is short-circuited. Then, the polarizer layer is a LHCP convertor. On the contrary, for a RHCP convertor, the MEMS switches have state \{“on", "off"\}. The two states represent two phase shifts with $180^{\circ}$ phase difference. The both states can be controlled by the metal pin via the inner ring with positive or negative DC voltage, moreover, the DC grounding can be easily achieved by the patch connecting to ground.

The patch of the reflection layer is a cross-shaped hollow ring with four symmetrically embedded MEMS elements, as shown in Fig. 5 (c). The completely symmetrical structure with respect to the coordinate axes is designed to compensate phase for the incident $\mathrm{CP}$ wave in a broad frequency band. Thus, four MEMS switches are operating simultaneously to obtain two resonant statuses with the state "off" and "on". By adjusting the position of the MEMS switches along the hollow ring, the reflection phase difference can be optimized as $90^{\circ}$ between the two states. The design process of the reflection layer has been demonstrated in [29], and the optimization works of the geometrical parameters are applied for the desired frequency band and phase shifts in our design. The biasing lines in red color in Fig. 5 (c) connect the central control metal pin with the four MEMS switches, and the biasing lines in green color are used for DC grounding of the switches. It is worth noticing that the green biasing grid is isolated from the metal patch via a $0.4 \mu \mathrm{m}$ thick silicon oxide layer (grey color in Fig.5 (a)) to avoid the short-circuit. Thus, the states of all the switches are controlled conveniently by the central control point with positive or null DC voltage.

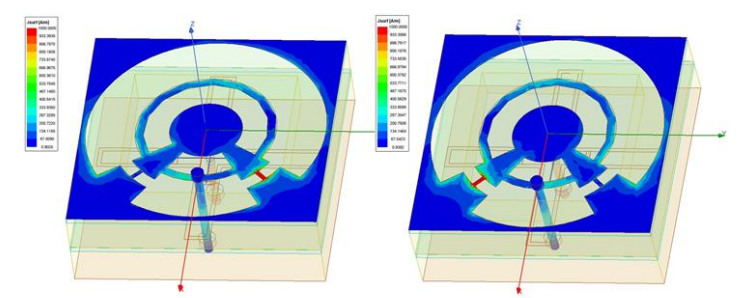

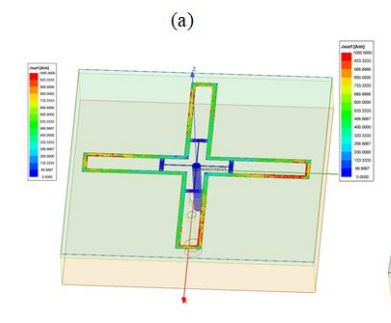

(c)

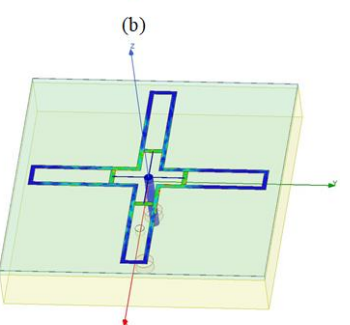

(d)
Fig. 6. Current distributions on the proposed RRA cell of different MEMS elements statuses. (a) state \{“off", "on"\} on the polarizer layer. (b) state \{“on", "off" $\}$ on the polarizer layer. (c) off-state on the reflection layer. (d) on-state on the reflection layer.

In summary, the combination of the polarizer layer and the reflection layer leads to four phase statuses. The heights of the Rohacell foams H1, H2 and H3 are optimized to obtain good impedance matching performance for all states of the unit cell. The simulation of the unit cell employs the Floquet port and the periodic boundary condition in HFSS. The packing effect of the applied MEMS switches is equivalent to the nominal on-state contact resistance and off-state capacitance of $0.9 \Omega$ and $15 \mathrm{fF}$, respectively, which are modeled in the full-wave simulation. The size of the MEMS switch is $230 \mu \mathrm{m} \times 100 \mu \mathrm{m} \times 5 \mu \mathrm{m}$, which allows the operation with low loss and high isolation performance up to $40 \mathrm{GHz}$. The MEMS switches are biased by utilizing high-resistivity polysilicon lines with a width of $15 \mu \mathrm{m}$ and resistance of $1.6 \mathrm{k} \Omega$. The effect of the bias lines on the device EM performance was also verified, where the same results are obtained with the bias lines presence or absence. The actuation voltage of the employed MEMS switches is in the range of $50 \mathrm{~V}$ and $80 \mathrm{~V}$.

The current distributions on the patches of the proposed unit cell at different states of the MEMS switches are shown in Fig. 6 . The distributions corresponding to the different resonances are switched by the MEMS elements to realize the 2-bit phase shift. Fig. 7 illustrates the simulated scattering parameters including the magnitude and phase of the active unit cell with all states. It can be concluded from the figure that the impedance bandwidth of the 2-bit unit cell with $S_{11}<-10 \mathrm{~dB}$ is from 29 to $30.4 \mathrm{GHz}$. The four phase response curves show good linearity to obtain smooth frequency variation. Moreover, the phase curves of the states " 00 " and " 10 " exhibit the same slope, as well as the curves of the states " 01 " and " 11 ". The consistencies are due to the tunable polarization approach that is irrelevant to the frequency. Therefore, the impedance and phase bandwidth can be improved by the optimization of the reflection layer in the future work. 


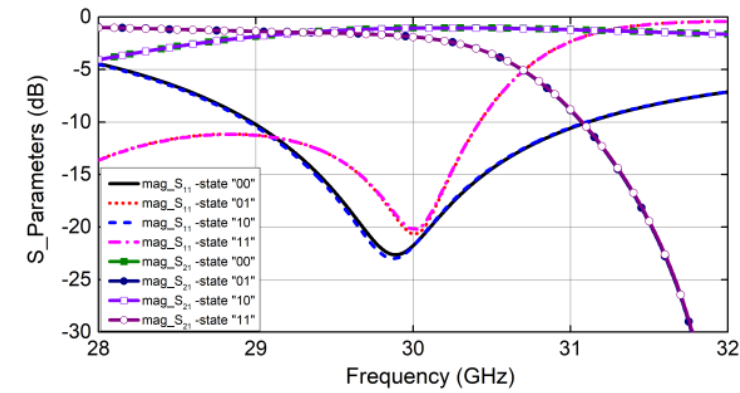

(a)

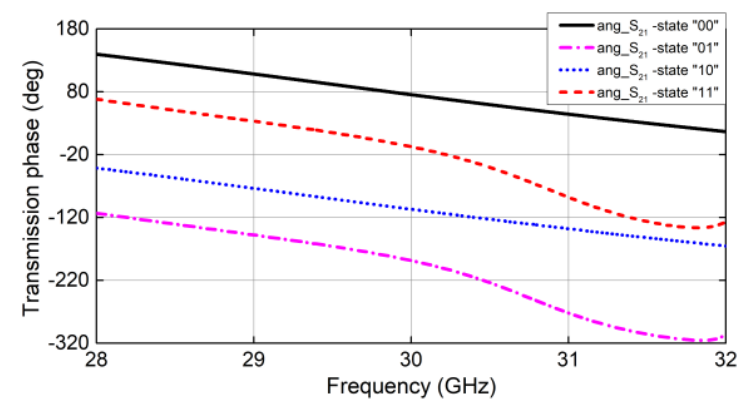

(b)

Fig. 7. Simulated scattering parameters (a) magnitude and (b) phase of the proposed 2-bit phase-resolution unit cell.

\section{B. Leaky-Wave Unit Cell for the Feed}

A conventional leaky-wave antenna consists of a partially reflective surface (PRS) and a metal ground plane separated by half-wavelength to form a leaky-wave cavity. In our design, the ground plane is replaced by a band-stop frequency selective surface (FSS) with a polarization selective function. The PRS and FSS are all periodical metal dipoles arrays printed on $0.254 \mathrm{~mm}$ Rogers RT/Duroid 5880 substrates separated by Rohacell foams. Partially and fully reflection can be achieved when the LP wave parallels to dipoles of the PRS and FSS, respectively, and both are transparent for the vertical LP wave. To integrate the feed with the reflectarray in a multi-layer PCB structure, the design and analysis of the leaky-wave structure are based on the same aperture as the unit cell. The layout of the leaky-wave unit cell is shown in Fig.8, where the geometrical parameters are given in the figure caption.

From [30], we can conclude that increased leakage efficiency and bandwidth can be obtained when the reflection coefficient remains constant in magnitude and a linear variation in phase response with the frequency variation of the PRS. On the other hand, the transparency of the orthogonal polarization wave is decided by the width and period of the PRS dipoles. Therefore, the geometrical parameters of the PRS dipoles are designed and optimized to obtain compromised performance of both the reflection and transmission modes. The simulated magnitude and phase of the reflection coefficients for the parallel $(\mathrm{S}(1: \mathrm{a}$, $1: a))$ and perpendicular ( $\mathrm{S}(1: \mathrm{b}, 1: \mathrm{b}))$ polarizations are plotted in Fig.9 (a). The magnitudes are $-2.5 \mathrm{~dB}$ and $-18 \mathrm{~dB}$, respectively. Moreover, the phase of the reflection coefficient follows linear variation for the parallel polarization. Therefore, partial reflection and good transmission are obtained for parallel and

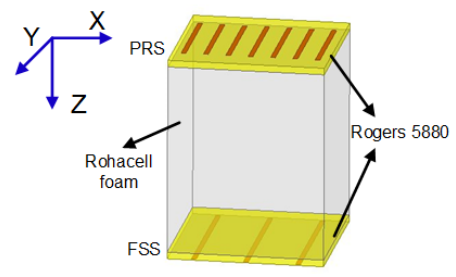

(a)

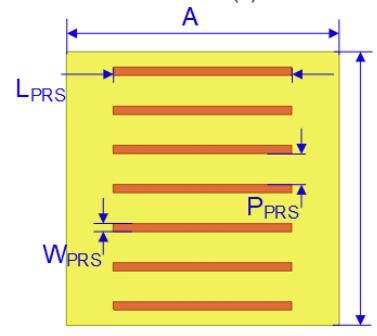

(c)

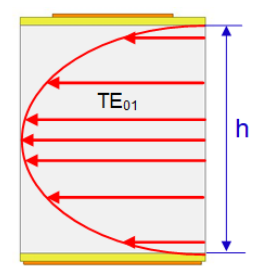

(b)

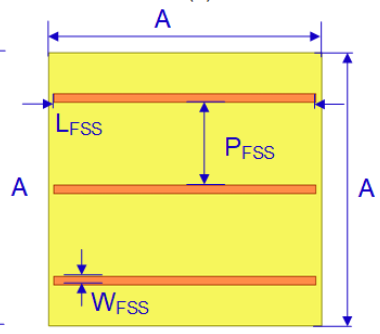

(d)
Fig. 8. The proposed leaky-wave structure. (a) Configuration. (b) Side view of 3D model. (c) Geometry of the PRS. (d) Geometry of the FSS. $\mathrm{h}=7.14 \mathrm{~mm}$, $\mathrm{A}=5.85 \mathrm{~mm}, \mathrm{~L}_{\mathrm{PRS}}=3.83 \mathrm{~mm}, \mathrm{~W}_{\mathrm{PRS}}=0.18 \mathrm{~mm}, \mathrm{P}_{\mathrm{PRS}}=0.66 \mathrm{~mm}, \mathrm{~L}_{\mathrm{FSS}}=5.63 \mathrm{~mm}$, $\mathrm{W}_{\mathrm{FSS}}=0.18 \mathrm{~mm}, \mathrm{P}_{\mathrm{FSS}}=1.77 \mathrm{~mm}$.

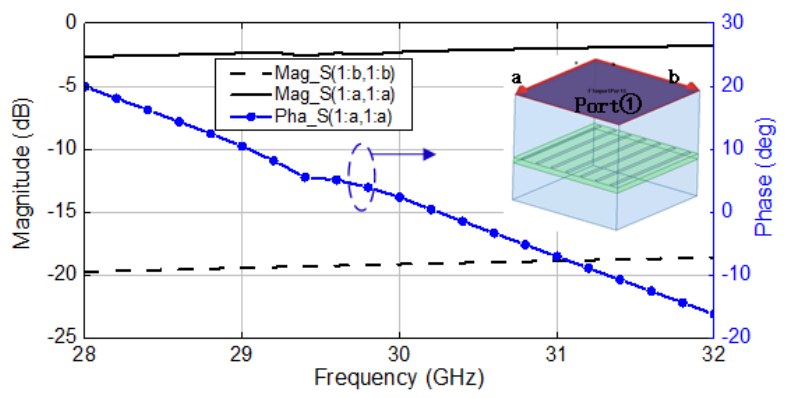

(a)

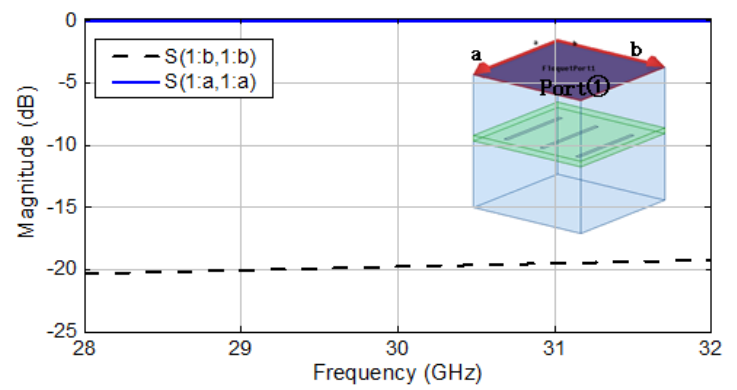

(b)

Fig. 9. Simulated reflection coefficients for the parallel $(S(1: a, 1: a))$ and perpendicular (S(1:b, 1:b)) polarizations. (a) PRS. (b) FSS.

perpendicular wave, respectively.

In the FSS design, the width of the FSS dipoles is identical to that of PRS for operating at the same resonant frequency. The length of the dipoles is approximately half wavelength to perform as a perfect electric conductor (PEC) for the parallel wave. To increase the transparency for the vertical wave, a larger period is selected as $1.77 \mathrm{~mm}$. The simulated reflection coefficients for the parallel and perpendicular polarizations of the FSS are plotted in Fig.9. (b). It can be observed that the return loss of the parallel wave is lower than $0.05 \mathrm{~dB}$, and higher than $18 \mathrm{~dB}$ for the vertical wave.

Based on the leaky-wave theory, the LM travels in the cavity 


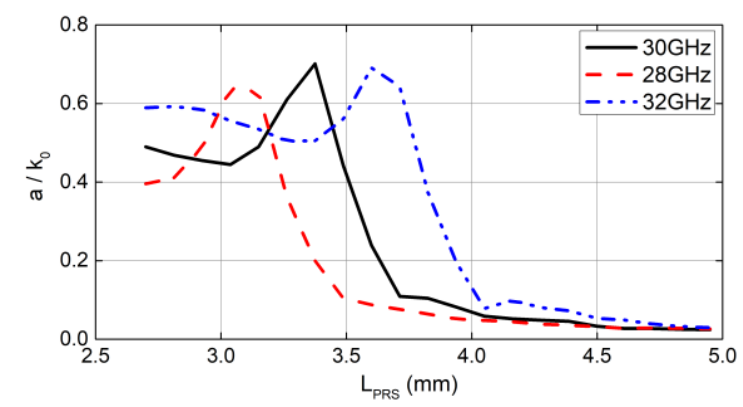

Fig. 10. Normalized leaky rate dispersion curves for different lengths of the PRS dipoles.



Fig.11. Simulated scattering parameters of the designed leaky-wave structure under feeding and transparent modes.

along the $\mathrm{x}$-direction with the complex constant $k=\beta$-j $\alpha$, where the $\beta$ is phase constant and $\alpha$ is leakage rate. Theoretically, the leakage rate determines the leakage efficiency by the equation of $\eta_{L}=1-e^{2 \alpha L}$. It can be concluded that the leakage rate is inversely proportional to the array length $(\mathrm{L}=\mathrm{NA})$ when the efficiency keeps constant. Moreover, for the large-scale array, a low leakage rate is preferred to decrease the taper magnitude aperture distribution along the LM propagating direction. Thus, it is required to control the leakage rate for different scale arrays to obtain compromise performance of leakage efficiency and relatively even magnitude distribution. As shown in [31], the length of PRS dipoles can be used to control $\alpha$. In our design, the normalized leakage rate curve $\left(\alpha / k_{0}\right)$ for different lengths ( $\mathrm{L}_{\mathrm{PRS}}$ ) is illuminated in Fig. 10 from the full-wave simulation. The curves at different frequencies disperse at the high leakage rate region but converge with the increasing $L_{\text {PRS. Therefore, it }}$ can be concluded that the increased radiation bandwidth of the leaky-wave feed is achieved for the large-scale array.

To comprehensively evaluate the performance of the leaky-wave structure, two different simulation methods with the master-slave boundary for transmission mode and radiation boundary for feeding mode are implemented, and the results are reported in Fig.11. The magnitudes of the reflection and transmission coefficients for the vertical polarization $(\mathrm{S}(1: \mathrm{b}$, $1: b)$ and $S(1: b, 2: b))$ under transmission mode are lower than $-20 \mathrm{~dB}$ and $0.5 \mathrm{~dB}$ from $28 \mathrm{GHz}$ to $32 \mathrm{GHz}$, respectively. For the LM wave, the reflection coefficient $(\mathrm{S}(1: 1,1: 1))$ is lower than $-20 \mathrm{~dB}$. Therefore, the above results show better impedance matching performance for the incident wave and reflected wave from the reflectarray plane.

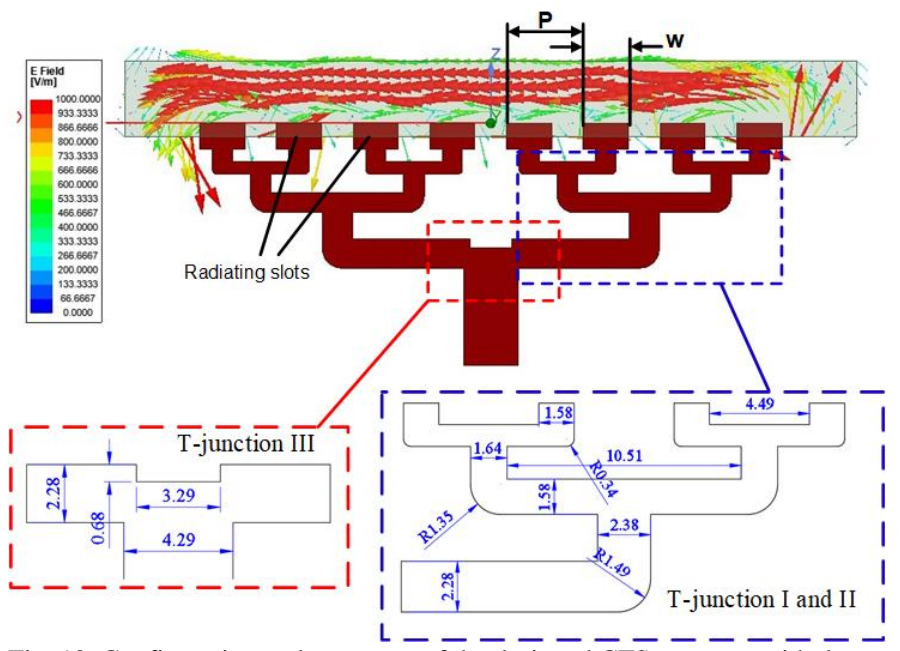

Fig. 12. Configuration and geometry of the designed $\overline{\mathrm{CTS}}$ structure with the distribution of electric field vector above the aperture.

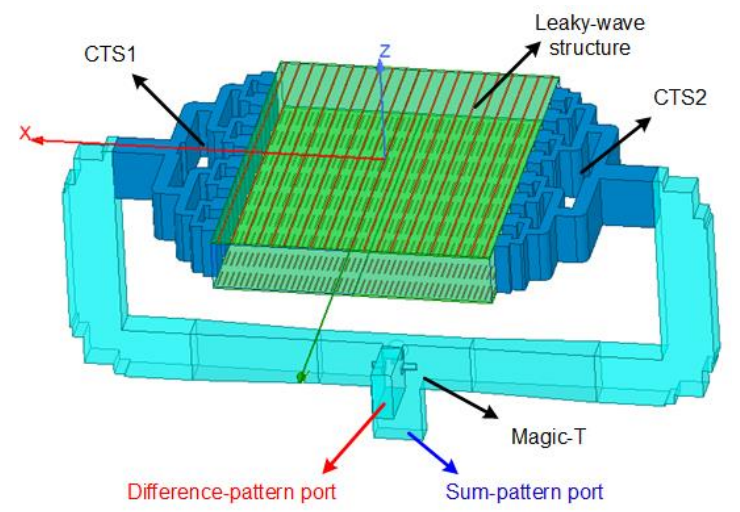

Fig. 13. Configuration of the proposed CTS-leaky-wave feed with magic-T and the definition of the ports.

\section{CTS Structure for Quasi-Plane Wave Excitation}

The CTS array is used to excite leaky-wave structure with a quasi-plane wave in order to obtain higher aperture efficiency and a more compact structure compared with taper-leaky-wave feed in [9]. Moreover, it can provide the design freedom on magnitude distribution in the y-direction to adapt different scale arrays for achieving low side-lobe levels. Because the power in the leaky-wave structure will emerge an exponential decay from the excitation end to the other end for unilateral excitation, the excitations from both sides are implemented to reduce the taper power distribution in the $\mathrm{x}$-direction.

Each CTS is an 8-slot array to adapt the scale of the proposed $9 \times 7$ reflectarray. The configuration and geometrical parameters of the designed CTS array are shown in Fig.12. The stub spacing $P$ is initially set to half wavelength at $30 \mathrm{GHz}$, and then optimized to be $6.075 \mathrm{~mm}$ for obtaining an optimal compromise including excitation efficiency, impedance matching, and structural adaptability to the leaky-wave cavity. The waveguide widths of the T-junctions from the common port to dividing ports decrease, then a natural impedance transformer is formed. The $90^{\circ}$ bends are designed with fillets on the outside edges to obtain good impedance matching performance over a wide frequency band. The simulated radiating electric field vector in 


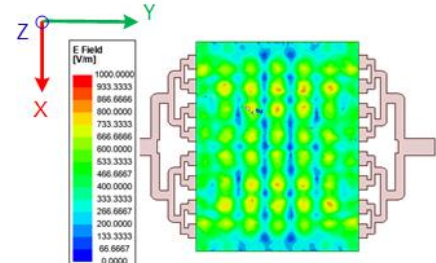

(a)

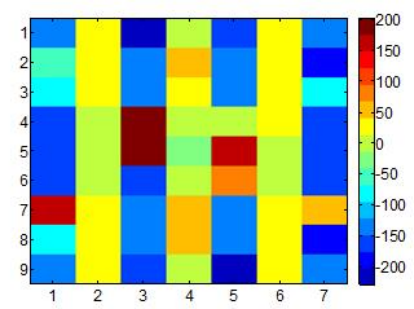

(c)


(b)

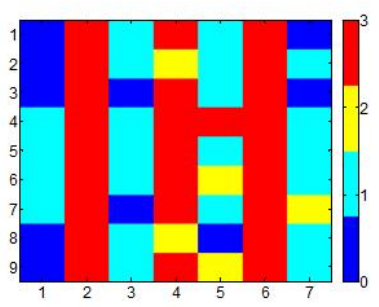

(d)
Fig. 14. (a) Simulated magnitude distribution on the proposed feed aperture. (b) phase distribution on the array aperture. (c) The incident phase distribution on the array aperture responding to each unit cell. (d) Phase control pattern in four discretized states for the main beam at broadside.

y-polarization on the aperture is also shown in Fig.12, equal magnitudes and phase wavefront are observed.

\section{Feed Array}

Fig. 13 shows the 3-D model and configuration of the proposed CTS-leaky-wave feed. Apart from the CTS and the leaky-wave structure, a waveguide magic- $T$ is utilized to provide two independent ports for the sum and difference beams. A metal post and a septum are employed in the magic-T design for impedance matching. The simulated magnitude and phase distribution on the feed aperture are plotted in Fig. 14 (a) and (b), respectively. It is worth noticing that the magnitude follows symmetric distribution in both the E-plane and H-plane, and the phase shows that the LM propagating in the leaky-wave cavity with the plane-wave form.

\section{LOW-Profile ReflectarRay Design AND SimUlation}

\section{A. Design Process}

Based on the above investigation, the presented reflectarray can be designed with the feed array suspended directly above the reflectarray plane. The distance between the feed and the plane should be as small as possible to reduce the profile and space loss. However, the mutual coupling between the feed and array increases with decreased distance, affecting the polarization rotation and leading to reduced radiation efficiency. Therefore, it is selected as $2 \mathrm{~mm}$ for optimal results.

For the different feeding mechanisms and operation principles, the conventional reflectarray synthesis method by calculating the optical path difference from the phase center of the feed to unit cells for obtaining the phase distribution on the reflectarray plane is not applicable in our design. Nevertheless, we can acquire the continuous phase distribution of the incident field on the array aperture from the full-wave simulation of the feed array, as shown in Fig.14 (b). It is worth pointing out that the extracted phase from the feed array is identical to that of the excitation field from the simulation of the whole reflectarray. Although the boundary conditions are different for the absence and presence of the reflectarray plane, the orthogonal polarizations between the incident and reflected wave lead to the same result of the excited-field phase distribution. Therefore, the polarization rotation is the basis of the beam synthesis method of the proposed reflectarray, otherwise, it is difficult to distinguish the excited-field phase from the aperture field on the reflectarray plane. The excited-field phase corresponding to each unit cell is plotted in Fig.14 (c), and whose distribution follows the plane-wave characteristic.

To focus the main beam in the direction $\left(\theta_{0}, \varphi_{0}\right)$, the delayed phases for each unit cell can be calculated by

$$
\begin{array}{r}
\varphi\left(x_{i}, y_{j}\right)=k_{0}\left(x_{i} \cos \varphi_{0}+y_{j} \sin \varphi_{0}\right) \sin \theta_{0} \\
-\varphi_{\text {Feed }}\left(x_{i}, y_{j}\right)+\Delta \varphi
\end{array}
$$

where $\varphi_{\text {Feed }}\left(x_{i}, y_{j}\right)$ is the discrete phase on the reflection layer from the feed at $\left(x_{i}, y_{j}\right), \Delta \varphi$ is a constant phase for further optimization [29] of the beams.

Because the calculated $\varphi\left(x_{i}, y_{j}\right)$ is the continuous phase shifts from (4), so which are discretized by $90^{\circ}$ to match the proposed unit cell of 2-bit phase quantization. The delayed phases for the main beam direction $\left(\theta_{0}, \varphi_{0}\right)=\left(0^{\circ}, 0^{\circ}\right)$ are calculated and shown in Fig.14 (d) with the $0 \sim 3$ responding to mode "00" to "11".

\section{B. Beam-Steering Performance and Bandwidth}

To efficiently validate the above beam synthesis method in our design process and explore the beam-steering performance, a passive design of the reflectarray with $9 \times 7$ unit cells is designed and simulated using a full-wave simulator with the MEMS elements replaced by the metal-line connections, and then the "On/Off" function can be simulated by the presence and absence of the metal-lines. The simulated steering beams in the E-plane are plotted in Fig. 15 (a) from $-30^{\circ}$ to $30^{\circ}$ with $15^{\circ}$ step at $30 \mathrm{GHz}$, and the sum and difference steering beams at $0^{\circ}$, $-15^{\circ}$ and $-30^{\circ}$ in the H-plane are shown in Fig. 15 (b). It can be seen that the simulated beams are pointing to the desired angles, especially deep-null difference beams can also steer to the desired angles with the sum beams in the H-plane. Therefore, the algorithm of the control code matrixes is verified. The simulated SLL is larger than $14.3 \mathrm{~dB}$ when the beam points at $0^{\circ}$, which is $6.1 \mathrm{~dB}$ and $9.9 \mathrm{~dB}$ for the main beam at $-30^{\circ}$ in the E-plane and H-plane, respectively. The high SLL is derived from the reverse-taper magnitude distribution from the feed and the phase error in phase digitalizing of the array. The simulated cross-polarization level is better than $25 \mathrm{~dB}$ in the main beam area of $-30^{\circ}$ to $30^{\circ}$, as shown in Fig. 15 (a). The simulated scanning loss is $2.4 \mathrm{~dB}$ and $3 \mathrm{~dB}$ in the E-plane and H-plane, respectively, endowing a 2-D beam-steering ability

The simulated gain and aperture efficiency of the $9 \times 7$ array with the function of frequency are plotted in Fig.16. The curves show that the gain difference is lower than $0.25 \mathrm{~dB}$ from $28 \mathrm{GHz}$ and $32 \mathrm{GHz}$, and the aperture efficiency is larger than $20 \%$ over the entire frequency range. 


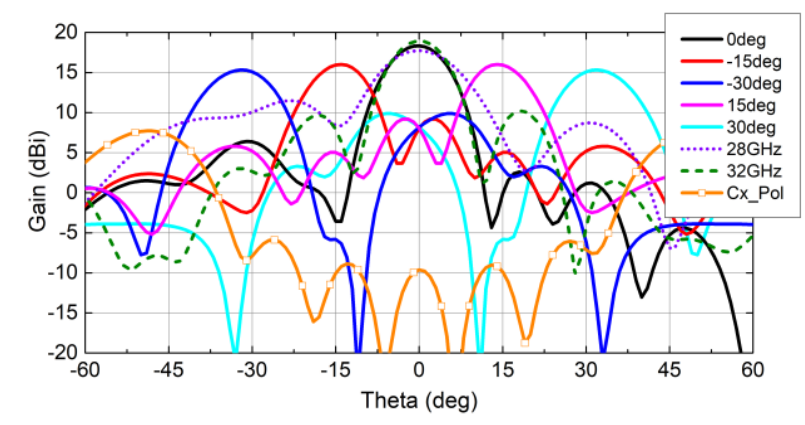

(a)

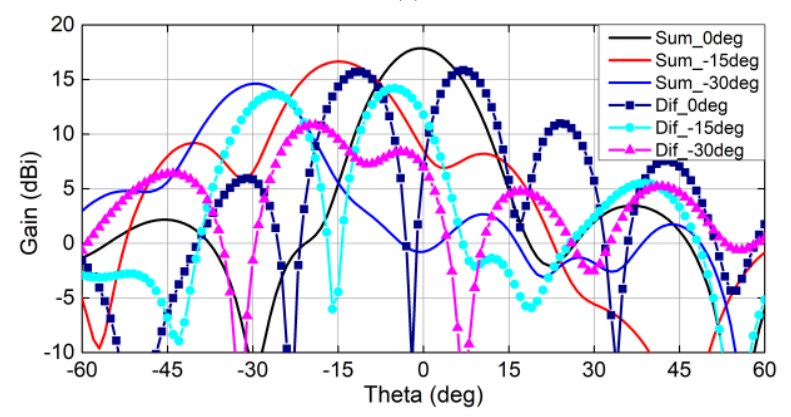

(b)

Fig. 15. The simulated scanning radiation patterns of the designed passive $9 \times 7$ reflectarray. (a) H- plane. (b) E-plane.

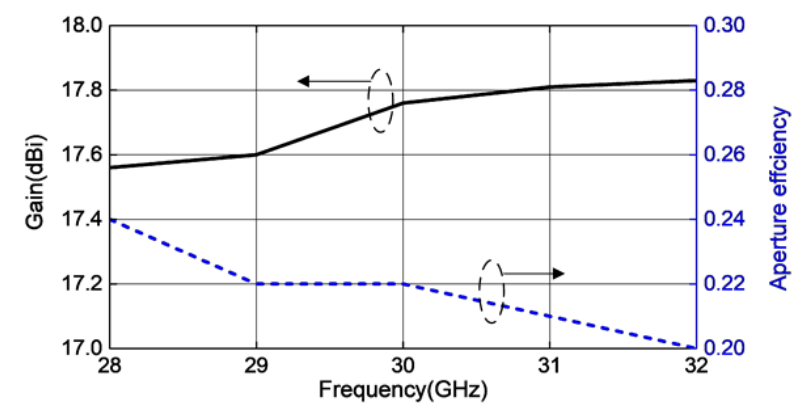

Fig. 16. The simulated gain and aperture efficiency of the $9 \times 7$ array.

\section{PRototyPe FABRICATION AND MEASUREMENT}

\section{A. Fabrication}

To fulfill the gain requirement of the application background, a passive prototype of the proposed low-profile reflectarray with $9 \times 67$ unit cells was designed, fabricated and measured for validation. The prototype is assembled by multilayer-PCB and metallic components. The metallic components including CTS, magic- $\mathrm{T}$, connecting waveguides and some brackets were manufactured in aluminium using milling and wire-cutting techniques. Both CTSs and the linked metallic cavity without top cover were machined and assembled as a union for providing the ground plane and two conductive boundaries to the multilayer PCB component. The PCB components composed of 2-bit unit cells array and leaky-wave structure were fabricated by the PCB lithography technology and adhesive based processes. The aperture of the entire prototype is $392 \times 54 \mathrm{~mm}^{2}\left(39.2 \lambda_{0} \times 5.4 \lambda_{0}\right)$, and the thickness without the magic-T is $12 \mathrm{~mm}\left(1.2 \lambda_{0}\right)$. The prototype is shown in Fig.17.

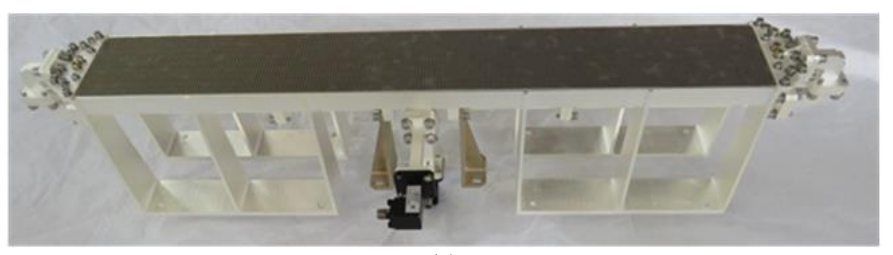

(a)

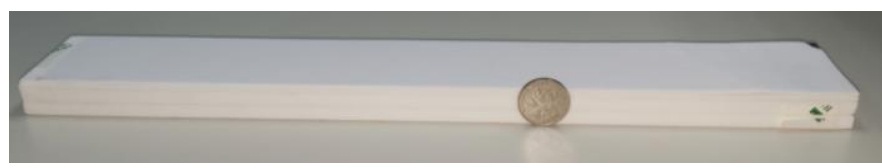

(b)

Fig. 17. Prototype of the proposed low-profile reflectarray. (a) Entire antenna. (b) Muilti-PCB component of leaky-wave structure and reflectarray plane.

\section{B. Measured Results and Discussion}

The measured S-parameters on the sum port and difference port of the waveguide magic-T of the prototype are shown in Fig.18 (a). The measured reflection coefficients of the two ports are lower than $-10 \mathrm{~dB}$ from $29.5 \mathrm{GHz}$ to $30.5 \mathrm{GHz}$, and the isolation between the two ports is higher than $17 \mathrm{~dB}$.

The radiation performance was measured by using a planar near-field system in an anechoic chamber. The measured and simulated results are compared and shown in Fig.18 (b)-(d). It can be observed that the main lobes of both E-plane and $\mathrm{H}$-plane co-polar patterns are consistent, except the measured gain is slighter lower than simulation. Due to the environmental reflection from the measuring system, the measured side lobes are asymmetrical, which are higher in the negative degree region than that of the positive degree region in both E- and H-plane. However, we can infer that the real SLL is higher than $8 \mathrm{~dB}$ from the comparison of the simulated result. Due to the fabrication and test errors, the measured cross-polarization level is higher than that of simulation. However, the measured results are below $-28.2 \mathrm{~dB}$ in both E-plane and $\mathrm{H}$-plane patterns. In terms of the difference pattern, the deviations between the test and simulation are the null position, null depth and lobe symmetry, which is mainly caused by the misalignment of the array and the probe of the near-field system. The measured null-depth is $-24.7 \mathrm{~dB}$, and the lobe difference is $1.29 \mathrm{~dB}$.

\section{Efficiency}

Table II lists the radiation performance of the sum beam, losses of separated components and calculated efficiencies of the prototype. The measured directivity at $30 \mathrm{GHz}$ of sum beam is $29.6 \mathrm{dBi}$, corresponding to the aperture efficiency of $35.1 \%$. The losses are derived from the phase, taper and spillover. The theoretical phase quantization loss is $0.91 \mathrm{~dB}$ for the 2-bit phase quantization, corresponding to the phase resolution efficiency of $88.3 \%$. The rest is $3.59 \mathrm{~dB}$ loss caused by taper magnitudes and spillover, which implies an illumination efficiency of $43.4 \%$. It is worth noticing that the result is excellent for a large-scale array with an extremely low profile, and it is predicted to be higher for a small array. Therefore, the proposed feed is proved as a highly efficient feeding mechanism with a low profile for a reflectarray. 


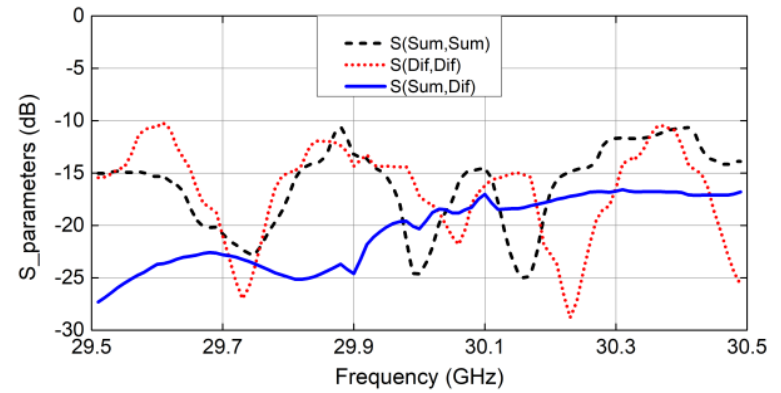

(a)

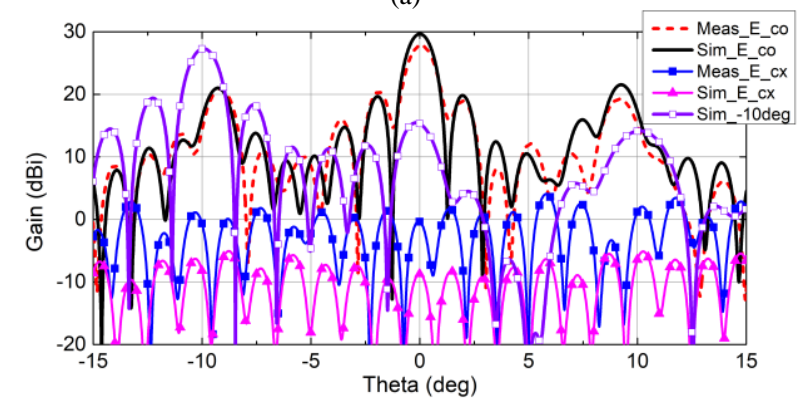

(b)

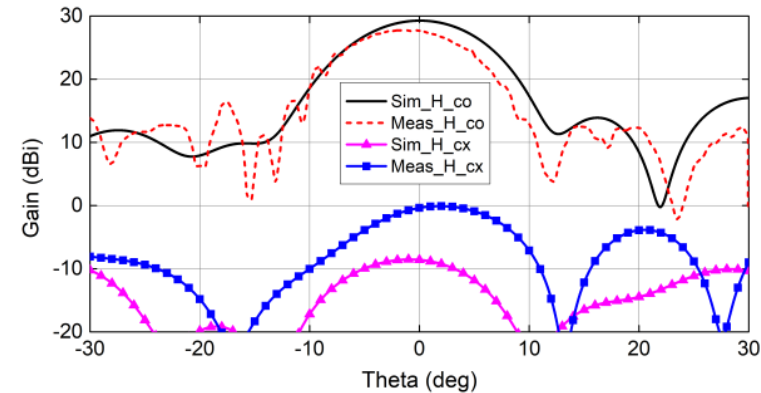

(c)

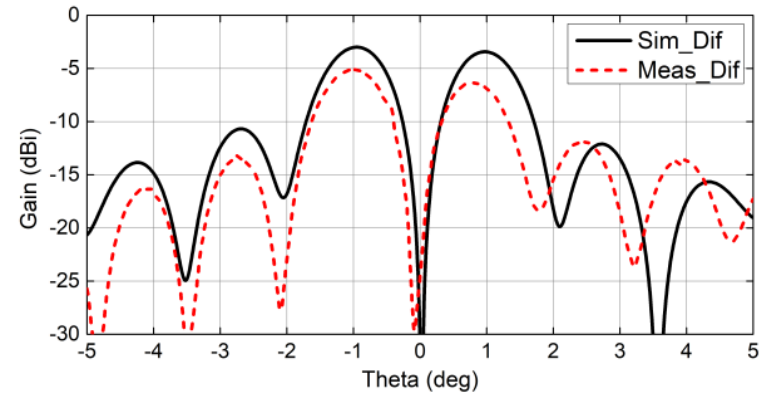

(d)

Fig. 18. (a) Measured scattering parameters in the ports of the magin-T. (b) Simulated and measured radiation patterns in the E-plane at $30 \mathrm{GHz}$. (c) Simulated and measured radiation patterns in the $\mathrm{H}$-plane at $30 \mathrm{GHz}$. (d) Simulated and measured difference patterns in the E-plane at $30 \mathrm{GHz}$.

The total efficiency including all losses was $23.2 \%$. The radiation efficiency is calculated as high as $79 \%$ from the comparison of the realized gain to the measured directivity, and the difference is the loss of the reflection layers. Moreover, the losses of mismatch and feed are estimated from the comparison of the realized gain and measured gain. Due to the low loss of the waveguide CTS structure, the loss of the whole feed is only $0.75 \mathrm{~dB}$, which is comparable to the horn feed of conventional front-fed reflectarray in Ka-band. Although the total efficiency is likely low, the aperture efficiency is still higher than the most
TABLE II

SUMMARY OF SOME RADIATION PERFORMANCE AND EFFICIENCIES OF THE DESIGNED PROTOTYPE

\begin{tabular}{cccccc}
\hline \hline \multicolumn{2}{c}{$\begin{array}{c}\text { Directivities } \\
\text { / Gains (dBi) }\end{array}$} & \multicolumn{2}{c}{ Losses (dB) } & \multicolumn{2}{c}{ Efficiencies } \\
\hline $\begin{array}{c}\text { Theoretical } \\
\text { Directivity }\end{array}$ & 34.1 & Phase & 0.91 & Phase resolution $\left(\varepsilon_{\mathrm{p}}\right)$ & $81.1 \%$ \\
\hline $\begin{array}{c}\text { Measured } \\
\text { Directivity }\end{array}$ & 29.6 & $\begin{array}{c}\text { Taper } \& \\
\text { spillover }\end{array}$ & 3.59 & Illumination $\left(\varepsilon_{\mathrm{i}}\right)$ & $43.4 \%$ \\
\hline $\begin{array}{c}\text { Realized } \\
\text { gain }\end{array}$ & 28.6 & $\begin{array}{c}\text { Reflection } \\
\text { layers }\end{array}$ & 1 & Radiation $\left(\varepsilon_{\mathrm{rd}}\right)$ & $79.4 \%$ \\
\hline $\begin{array}{c}\text { Measured } \\
\text { gain }\end{array}$ & 27.8 & $\begin{array}{c}\text { Mismatch } \\
\text { Feed Loss }\end{array}$ & 0.05 & Mismatch $\left(\varepsilon_{\mathrm{r}}\right)$ & $99 \%$ \\
\hline & Total & Aperture $\left(\varepsilon_{\mathrm{ap}}=\varepsilon_{\mathrm{p}} \varepsilon_{\mathrm{i}}\right)$ & $35.1 \%$ \\
\hline \hline
\end{tabular}

existing demonstrations of 1-bit reflectarray [11-13].

\section{Scalability}

Since the simulations of the unit cells in section III are based on the periodic boundary to imitate an infinite array, then the scaling of the reflectarray can be easily performed without deterioration in the performance. Generally, for the large array, the phase errors will be averaged over a large number of unit cells, thus a single cell has less impact on the array. Therefore, the large array will have better radiation and scanning performance theoretically compared with a small array.

In terms of the feed array, the scaling in the lateral direction can be achieved by employing more leaky-wave columns, a larger-scale CTS array and more cascaded power dividers. Because the increased scale of waveguide CTS will not enlarge the loss proportionally, so the improved efficiency can be obtained at the price of the bulky structure. In the leaky-wave propagation direction, the scaling can be realized by controlling the leakage rate as discussed in section III. Therefore, the illumination efficiency will not be affected by the array scale.

Some of the above conclusions can be certified in our work by the comparison of simulation results of $9 \times 7$ array and $9 \times 67$ array shown in Fig. 16 and Fig. 19, respectively.

\section{E. Comparison}

In recent years, some demonstrations on low-profile RA or 2-bit RA are reported. However, to our best knowledge, no existing work has both the above features. Therefore, to evaluate the performance of the presented reflectarray antenna, we compare the measured results with those low-profile or 2-bit reflectarray / transmittarray reported in literature, as shown in Table III. Compared to the FRA in [3], conventional offset-fed [19-20] and front-fed [21] 2-bit RAs, the aperture efficiency of our design is comparable but the height is significantly reduced in our design. Moreover, the height of the RA does not increase with the enlarged aperture. The transmittarray integrated with leaky-wave feed introduced in [9] shows similar height and efficiency based on a small array prototype with a continuous phase shift. However, the waveguide taper feed occupies a huge space, and the efficiency will also be decreased with the increased scale. In addition, tracking function for the RRA is 
TABLE III

COMPARISON OF 2-BIT OR LOW-PROFILE REFLECTARRAY AND TRANSMITARRAY ANTENNAS

\begin{tabular}{cccccc}
\hline \hline Ref. & $\begin{array}{c}\text { Operation } \\
\text { Frequency }\end{array}$ & $\begin{array}{c}\text { Dimension } \\
(\mathbf{L} \times \mathbf{W} \times \mathbf{H})\end{array}$ & $\begin{array}{c}\text { Aperture } \\
\text { efficiency }\end{array}$ & $\begin{array}{c}\text { Feeding } \\
\text { Type }\end{array}$ & Bit Number \\
\hline$[3]$ & $29 \mathrm{GHz}$ & $\pi \times 8.1^{2} \lambda^{2} \times 3.5 \lambda$ & $37.2 \%$ & FRA & Continuous \\
\hline$[9]$ & $4.8 \mathrm{GHz}$ & $2.9 \lambda \times 2.9 \lambda \times 0.75 \lambda$ & $34 \%$ & $\begin{array}{c}\text { Leaky } \\
\text { wave TA }\end{array}$ & Continuous \\
\hline$[19]$ & $35 \mathrm{GHz}$ & $7 \lambda \times 6.4 \lambda \times 7 \lambda$ & $37.9 \%$ & Offset fed & 2 \\
\hline$[20]$ & $9.65 \mathrm{GHz}$ & $9.65 \lambda \times 9.65 \lambda \times \mathrm{NG}$ & $37.3 \%$ & Offset fed & 2 \\
\hline$[21]$ & $10 \mathrm{GHz}$ & $\pi \times 25 \lambda^{2} \times 8.5 \lambda$ & $39.4 \%$ & Front fed & 2 \\
\hline $\begin{array}{c}\text { This } \\
\text { work }\end{array}$ & $30 \mathrm{GHz}$ & $43 \lambda \times 5.4 \lambda \times 1.2 \lambda$ & $35.1 \%$ & $\begin{array}{c}\text { Leaky } \\
\text { wave RA }\end{array}$ & 2 \\
\hline \hline
\end{tabular}

obtained in the proposed work for the first time through the feeding mechanism. Although the tracking accuracy is not fascinating due to the 2-bit phase quantization error, which can be improved by employing the continuous phase shift design.

\section{CONCLUSION}

In this paper, we propose a novel antenna architecture to greatly reduce the profile of the beam-steering reflectarray. By utilizing two CTSs to excite a leaky-wave structure with a uniform plane-wave excitation, a highly efficient CTS-leaky-wave feed is realized. Moreover, a waveguide magic- $\mathrm{T}$ is used to provide in-phase and out-of-phase excitations for both CTSs, then obtaining sum and difference beams simultaneously. To realize the beam-steering and increase the aperture efficiency, a new unit cell with a 2-bit phase quantization and polarization rotation function is designed by using a new phase shift method that combines tunable polarization and discrete resonator. The 2-D beam scanning performance and wide gain bandwidth are verified by the full-wave simulation, and then a prototype with $9 \times 67$ unit cells is fabricated and measured. The measured results show a high illumination efficiency of the feed and a high aperture efficiency with the entire thickness of the prototype without magic-T is about $3 \%$ of that of a conventional reflectarray with the same aperture. Therefore, the proposed reflectarray antenna is an attractive and competitive candidate for satellite antennas.

\section{REFERENCES}

[1]J. Huang, J. A. Encinar, Reflectarray antennas, New Jersey, USA: IEEE-Wiley, 2008.

[2]D. Pilz and W. Menzel, "Folded reflectarray antenna," Electron. Lett., vol. 34, no. 9, pp. 832-833, Apr. 1998.

[3] Y. Cao, W. Q. Che, W. C. Yang, C. Fan and Q. Xue, "Novel wideband polarization rotating metasurface element and its application for wideband folded reflectarray," IEEE Trans. Antennas Propag., vol. 68, no. 3, pp. 2118-2127, Oct. 2019

[4] Q. Luo, S. Gao, C. Zhang, D. Zhou, T. Chaloun, W. Menzel, V. Ziegler and M. Sobhy, "Design and analysis of a reflectarray using slot antenna elements for Ka-band SatCom," IEEE Trans. Antennas Propag., vol. 63, no. 4, pp. 1365-1374, Apr. 2019.

[5]C. Zhang et al., "A planar integrated folded reflectarray antenna with circular polarization," IEEE Trans. Antennas Propag., vol. 65, no. 1, pp. 385-390, Jan. 2017.

[6]S.-W. Qu, H. X. Zhang, W.-W. Wu, P.-F. Li, S. Yang and Z. P. Nie, "Wideband folded reflectarray using novel elements with high orthogonal polarization isolation," IEEE Trans. Antennas Propag., vol. 64, no. 7, pp. 3195-3200, Jul. 2016.

[7]S. Bildik, S. Dieter, C. Fritzsch, W. Menzel and R. Jakoby, "Reconfigurable folded reflectarray antenna based upon liquid crystal technology," IEEE Trans. Antennas Propag., vol. 63, no. 1, pp. 122-132, Jan. 2015.

[8]J. Nicholls and S. V. Hum, "An electronically steerable reflectarray with integrated leaky-wave feed," in Proc. IEEE Int. Symp. Antennas Propag. USNC/URSI Nat. Radio Sci. Meeting, Jul. 2015, pp. 2175-2176.

[9]J. G. Nicholls and S. V. Hum, "Full-space electronic beam-steering transmitarray with integrated leaky-wave feed," IEEE Trans. Antennas Propag., vol. 64, no. 8, pp. 3412-3422, Aug. 2016.

[10] S. V. Hum and J. P. Carrier, "Reconfigurable reflectarrays and array lenses for dynamic antenna beam control: A review," IEEE Trans. Antennas Propag., vol. 62, no. 1, pp. 183-198, Jan. 2014.

[11] M. T. Zhang et al., "Design of novel reconfigurable reflectarrays with single-bit phase resolution for ku-band satellite antenna applications," IEEE Trans. Antennas Propag., vol. 64, no. 5, pp. 1634-1641, May 2016.

[12] H. Yang et al., "A 1-bit $10 \times 10$ reconfigurable reflectarray antenna: Design, optimization, and experiment," IEEE Trans. Antennas Propag., vol. 64, no. 6, pp. 2246-2254, Jun. 2016.

[13] H. Kamoda, T. Iwasaki, J. Tsumochi, T. Kuki and O. Hashimoto, "60-GHz electronically reconfigurable large reflectarray using single-bit phase shifters," IEEE Trans. Antennas Propag., vol. 59, no. 7, pp. 25242531, Jul. 2011.

[14] B. Wu, A. Sutinjo, M. E. Potter and M. Okoniewski, "On the selection of the number of bits to control a dynamic digital MEMS reflectarray," IEEE Antennas Wireless Propag. Lett., vol. 7, pp. 183-186, Jul. 25, 2008.

[15] R. Pereira, R. Gillard, R. Sauleau, P. Potier, T. Dousset and X. Delestre, "Dual linearly-polarized unit-cells with nearly 2-bit resolution For reflectarray applications in X-band," IEEE Trans. Antennas Propag., vol. 60, no. 12, pp. 6042-6048, Dec. 2012.

[16] X. Yang, S. Xu, F. Yang and M. Li, "A novel 2-bit reconfigurable reflectarray element for both linear and circular polarizations," 2017 IEEE Int. Symp. Antennas and Propagation, San Diego, CA, 2017, pp. 2083-2084.

[17] B. D. Nguyen, V. S. Tran, L. Mai, P. H. Dinh, "Two-bit reflectarray element using cut-ring patch coupled to delay lines," REV J. Electronics Commumications, vol. 6, no. 1-2, pp. 30-34, 2016.

[18] X. Yang, S. Xu, F. Yang and M. Li, "Design of a 2-bit reconfigurable reflectarray element using two MEMS switches," 2015 IEEE Int. Symp. Antennas and Propagation, Vancouver, BC, 2015, pp. 2167-2168.

[19] C. Chih-Chieh and A. Abbaspour-Tamijani, "Design and experimental verification of steerable reflect-arrays based on two-bit antenna-filter-antenna elements," in Proc. IEEE Int. Microwave Symp., Boston, MA, Jun. 2009, pp. 1181-1184.

[20] C. Apert, T. Koleck, P. Dumon, T. Dousset, and C. Renard, "ERASP: A new reflectarray antenna for space applications," presented at the 1st Eur. Conf. Antennas Propag, EuCAP, Nice, France, Nov. 6-10, 2006.

[21] H. Luyen, J. H. Booske, and N. Behdad, "2-bit phase quantization using mixed polarization-rotation/non-polarization-rotation reflection modes for beam-steerable reflectarrays," IEEE Trans. Antennas Propag., Early Access, Jun. 2020.

[22] P.Naseri et al, "A dual-band dual-circularly polarized reflectarray for K/Ka-band space applications," IEEE Trans. Antennas Propag., vol. 68, no. 6. Jun. 2020.

[23] P. Naseri et al, "Dual-band dual-linear-to-circular polarization converter in transmission mode application to K/Ka-band satellite communications," IEEE Trans. Antennas Propag., vol 66, no. 12. Oct. 2019.

[24] A. A. Oliner, "Leaky-wave antennas," Antenna Engineering Handbook, R. C. Johnson, Ed., 3rd ed. New York: McGraw-Hill, 1993, ch.10.

[25] R. G. Quirós, J. L. Tornero, A. R. Weily and Y. J. Guo, "Electronically steerable 1-D Fabry-Pérot leaky-wave antenna employing a tunable high impedance surface," IEEE Trans. Antennas Propag., vol. 60, no. 11, pp. 5046-5055, Nov. 2012.

[26] M. Ettorre, F. F. Manzillo, M. Casaletti, R. Sauleau, L. L. Coq, and N. Capet, "Continuous transverse stub array for Ka-band applications," IEEE Trans. Antennas Propag., vol. 63, no. 11, pp. 4792-4800, Nov. 2015.

[27] T. Potelon, M.Ettorre, L. Le Coq, T. Bateman, J. Francey, D. Lelaidier, E. Seguenot, F. Devillers, and R. Sauleau, "A low-profile broadband 32-slot continuous transverse stub array for backhaul applications in E-band," IEEE Trans. Antennas Propag., vol. 65, no. 12, pp. 6307-6316, Dec. 2017

[28] T. Potelon, M.Ettorre, L. Le Coq, T. Bateman, J. Francey, and R. Sauleau, "Reconfigurable CTS antenna fully integrated in PCB technology for 5G backhaul applications," IEEE Trans. Antennas Propag., vol. 67, no. 6, pp. 3609-3618, Jun. 2019. 
[29] T. Debogovic and J. P. Carrier, "Low loss MEMS-reconfigurable 1-bit reflectarray cell with dual-linear polarization," IEEE Trans. Antennas Propag., vol. 62, no. 10, pp. 5055-5060, Oct. 2014.

[30] J R. Kelly, T. Kokkinos, A. P. Feresidis, "Analysis and design of sub-wavelength resonant cavity type 2-D leaky-wave antennas," IEEE Trans. Antennas Propag., vol. 56, no. 9, pp. 2817-2825, Sep. 2008.

[31] M. García-Vigueras, J. L. Gómez-Tornero, G. Goussetis, A. R. Weily, and Y. J. Guo, "1D-leaky-wave antenna employing parallel-plate waveguide loaded with PRS and HIS," IEEE Trans. Antennas Propagat., vol. 59, no. 10, pp. 3687-3694, Oct. 2011.

[32] A. H. Abdelrahman, P. Nayeri, A. Z. Elsherbeni, and F. Yang, "Bandwidth improvement methods of transmitarray antennas," IEEE Trans. Antennas Propag., vol. 63, no. 7, pp. 2946-2954, Jul. 2015.

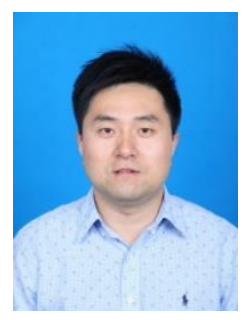

Qiaoshan Zhang received the B.S. and M.S. degrees in Beijing institute of technology, Beijing, China, in 2009 and 2012, respectively. He is currently working toward the Ph.D. degree with the Xidian University, Xi'an, China. From Oct. 2019 to Oct. 2020, he was a Visiting Scholar with the University of Kent, U.K. He is currently a Senior Engineer with Xi'an Institute of Space Radio Technology, Xi'an, China. His current research interests include satellite antennas, UWB antennas, and antenna arrays

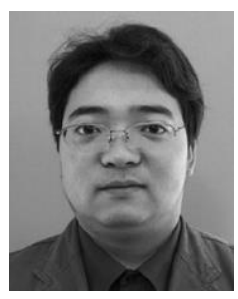

Ming-Tao Zhang (M'14) received the B.S. and M.S. degrees in electrical engineering from Xidian University, Xi'an, China, in 2004 and 2007, respectively.

He was a professor with Xi'an Institute of Space Radio Technology (XISRT), Xi'an, China. From 2013 to 2014, he was a Visiting Scholar with the University of Kent, Canterbury, U.K. His current research interests include mm-wave antennas, reflectarray, feed assembly, and satellite antenna design and verification.

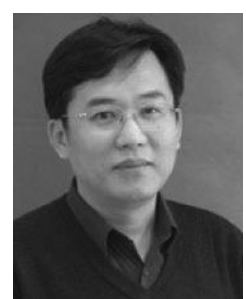

Xiao Wei Shi (SM'08) received the B.S. degree, M.E. degree and Ph.D. degree in electromagnetic field and microwave technology from Xidian University, Xi'an, China, in 1982, 1990, and 1995, respectively.

From 1996 to 1997, he was a Cooperator with the Electronics and Telecommunications Research Institute, Daejeon, South Korea, for his post-doctoral research work. He is currently a Professor and a Ph.D. Student Advisor with Xidian University. His research interests include the theory of microwave network, microwave measurement, electromagnetic inverse scattering, the theory of electromagnetic variation, electromagnetic compatibility, and smart antenna.

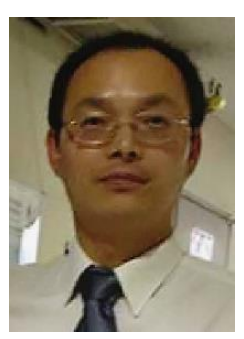

Steven Gao (M'01-SM'16) received the Ph.D. degree in microwave engineering from Shanghai University, Shanghai, China, in 1999.

He is currently a Professor and the Chair of RF and Microwave Engineering, University of Kent, U.K. His current research interests include smart antennas, phased arrays, MIMO, satellite antennas, satellite communications, UWB radars, synthetic aperture radars, and mobile communications. He is an Associate Editor of several journals including the IEEE Transactions on Antennas and Propagation.

Qi Luo (S'08-M'12-SM'19) is currently a Senior Lecturer with the School of Physics, Engineering and Computer Science, University of Hertfordshire, Hatfield, U.K.
Lei Chen is currently an Associate Professor with the Xidian University, Xi'an, China.

Jixiang Wan is currently a Professor and an Associate Director of the Space Antenna Department, Xi'an Institute of Space Radio Technology (XISRT), Xi'an, China.

Xudong Wang is currently a senior engineer of the Space Antenna Department, Xi'an Institute of Space Radio Technology (XISRT), Xi'an, China. 